\title{
Comparison of aggregation behaviors between branched and linear block polyethers: MesoDyn simulation study
}

\author{
Houjian Gong • Guiying Xu • Xiaofeng Shi • Teng Liu • \\ Zhiwei Sun
}

Received: 30 June 2010 /Revised: 22 August 2010 /Accepted: 2 September 2010 / Published online: 21 September 2010

(C) Springer-Verlag 2010

\begin{abstract}
The comparison of aggregation behaviors between the branched block polyether T1107 (polyether A) and linear polyether $(\mathrm{EO})_{60}(\mathrm{PO})_{40}(\mathrm{EO})_{60}$ (polyether $\mathrm{B}$ ) in aqueous solution are investigated by the MesoDyn simulation. Polyether A forms micelles at lower concentration and has a smaller aggregation number than B. Both the polyethers show the time-dependent micellar growth behaviors. The spherical micelles appear and then change to rod-like micelles with time evolution in the $10 \mathrm{vol} \%$ solution of polyether A. The micellar cluster appears and changes to pseudo-spherical micelles with time evolution in the $20 \mathrm{vol} \%$ solution of polyether A. However, the spherical micelles appear and change to micellar cluster with time evolution in the $20 \mathrm{vol} \%$ polyether B solution. The shear can induce the micellar transition of both block polyethers. When the shear rate is $1 \times 10^{5} \mathrm{~s}^{-1}$, the shear can induce the sphere-to-rod transition of both polyethers at the concentration of 10 and $20 \mathrm{vol} \%$. When the shear rate is lower than $1 \times 10^{5} \mathrm{~s}^{-1}$, the huge micelles and micellar clusters can be formed in the 10
\end{abstract}

Electronic supplementary material The online version of this article (doi:10.1007/s00396-010-2294-7) contains supplementary material, which is available to authorized users.

H. Gong $\cdot$ G. Xu $(\bowtie) \cdot$ X. Shi $\cdot$ T. Liu

Key Laboratory of Colloid and Interface Chemistry,

Ministry of Education, Shandong University,

Jinan 250100, People's Republic of China

e-mail: xuguiying@sdu.edu.cn

Z. Sun

Key Laboratory of Microgravity, Institute of Mechanics,

Chinese Academy of Sciences,

Beijing 100190, People's Republic of China

Z. Sun

National Microgravity Laboratory, Institute of Mechanics,

Chinese Academy of Sciences,

Beijing 100190, People's Republic of China and $20 \mathrm{vol} \%$ polyether A systems under the shear, while the huge micelles are formed and then disaggregated with the time evolution in the $20 \mathrm{vol} \%$ polyether B system.

Keywords Branched block polyether-Aggregation behavior $\cdot$ Micelle $\cdot$ MesoDyn simulation

\section{Introduction}

Poly(ethylene oxide)-poly(propylene oxide)-poly(ethylene oxide) (PEO-PPO-PEO) block polyethers are macromolecular-nonionic surfactants. The block polyethers can selfassemble due to the character of molecule with amphiphilic structure resembling that of the low-molecular-weight surfactants. In aqueous medium, the block polyethers exist in the molecular state at low concentration or temperature, while they do in the form of intermolecular micelles above the critical micellization concentration (CMC) or the critical micellization temperature [1-5]. The formation of micelle in aqueous block polyether solution has an important practical application in many industrial fields [6-9]. The aggregation behaviors of block polyethers have been studied extensively by various methods, for example, micro-calorimetry [10-12], small-angle neutron or X-rays scattering [13-15], static and dynamic light scattering [1618], and rheometry [19-22]. Besides, computer simulation methods, such as dissipative particle dynamics [23-25] and mesoscopic dynamics (MesoDyn) [26-28] have been used in the study on the aggregation behavior of polyether. These mesoscopic models build a bridge between fast molecular kinetics and slow thermodynamic relaxation of macro-scale properties [29]. MesoDyn simulation has led to significant advances in the investigation of the aggregation behaviors of block polyethers [30-32]. The information of 
simulation time of micelle formation, phase behavior of block copolymers, formation of mesoscale structures, and the solution behavior of specific chemical species can be gotten by MesoDyn simulation [30, 32].

Although the aggregation behaviors of the block polyethers are investigated in detail as a function of temperature and concentration, the studies of the time dependence in the micellar growth and restructuring process is difficult by means of experimental methods. The sphere-to-rod growth behavior of the triblock polyether $\mathrm{EO}_{20} \mathrm{PO}_{70} \mathrm{EO}_{20}(\mathrm{P} 123)$ has been observed; however, not all the triblock polyethers show the shape transition of their micelles in the experiment [33]. However, the time dependence of the micellar growth can be observed clearly in the MesoDyn simulation [34].

Recently, we have explored the properties of branched block polyethers and compared with the linear triblock polyethers [35-41]. The results show that branched block polyether has many advantages in practical applications, such as in synthesis of nanoparticles [36], oil demulsification [37, 38], and dispersing carbon nanotubes [39]. The aggregation behaviors of a branched block polyether (AP432) compared to the linear triblock polyether L64 at the air/water surface and in bulk aqueous solutions have been investigated $[40,41]$. We find that both the efficiency and the effectiveness of AP432 to lower the surface tension of water are higher than those of L64 and the micelles formed by AP432 are more compact than that of L64. Although the investigations show the advantage of the branched block polyethers, it is unclear that whether the micelle formation process of the branched block polyethers is different from that of the linear polyethers. Therefore, the MesoDyn simulation method is used to investigate the difference between the time dependence of micellar growth behaviors for the branched and linear block polyethers, and the effect of shear on the aggregation process. The aim is further to understand the difference of aggregation behaviors between the branched and linear block polyethers, and to provide useful information for their practical applications.

\section{Simulation methods}

The MesoDyn simulation is based on the dynamics density functional theory. The essential idea of the simulation method is that if the free energy of an inhomogeneous liquid is a function of the local density function, and then all thermodynamic functions can be derived [30-32, 42]. Various types of beads are used as the model in the MesoDyn simulations. Each bead is a certain component type representing covalently bonded groups of atoms, such as the hydrophobic or hydrophilic groups in the amphi- philes. The interaction between different beads is used to describe by harmonic oscillator potentials for the intramolecular interactions [43]. The dynamic functions of the system are described by the Langevin equations, which are the diffusion equations in the component densities taking account of the noise in the system. Therefore, to specify the chemical nature of the system in MesoDyn simulation, two sets of parameters have to be defined, one is for the Gaussian chain in terms of repeat beads and the other is for the interaction energies of the various components. For the first set, a Gaussian chain "spring and beads" description is used in the MesoDyn simulation. The Gaussian chain has the same size, and the chain topology depends on the coarsened degree of the original system.

Numerous works on the MesoDyn simulation of the block polyether have been carried out. Usually in the model, springs mimic the stretching behavior of a chain fragment and different kinds of beads correspond to different components in the block polyether. Each bead in the Gaussian chain is a statistical unit, representing a number of "real" monomers. Therefore, the choice of the Gaussian chain is an important aspect of the method. Van Vlimmeren et al. [44] obtained a simple relationship for atomistic and Gaussian chains:

$\frac{X}{x} \approx 4.3, \frac{Y}{y} \approx 3.3$

where $X$ and $Y$ refer to the numbers of units in the PEO and PPO block polymer, respectively, and $x$ and $y$ are the numbers of coarsened chain topology. However, since both $x$ and $y$ have to be an integer, the above equations cannot be exactly solved.

In this work, the branched block polyether Tetronic 1107 (polyether A) and the linear block polyether $(\mathrm{EO})_{60}(\mathrm{PO})_{40}(\mathrm{EO})_{60}$ (polyether B) are contrastively investigated by the MesoDyn simulation. The molecular structures of A and B are shown in Fig. 1. In the simulation, a few approximate values are chosen and the Gaussian chain scheme of polyether A is E 14 P 6 P [P 6 E 14] [P 6 E 14] P $6 \mathrm{E} 14$. While the Gaussian chain of polyether B is E $14 \mathrm{P}$ $12 \mathrm{E} 14$, where the $\mathrm{E}$ blocks represent the $\mathrm{PEO}$, the $\mathrm{P}$

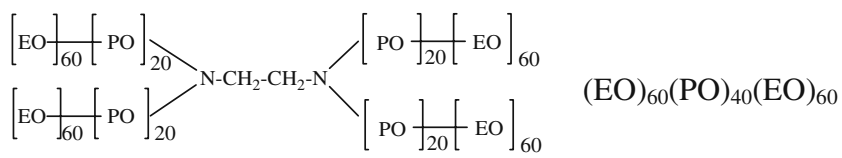

(A)

Fig. 1 The molecular structure of the branched and linear block polyethers. a Branched block polyether A, $M_{\mathrm{n}}=15,000 \mathrm{~g} / \mathrm{mol}$, the mass percentage of EO is $69.2 \%$; b the linear block polyether B, $M_{\mathrm{n}}=7,600 \mathrm{~g} / \mathrm{mol}$, the mass percentage of EO is $69.5 \%$ 
blocks are the PPO, and one bead $\mathrm{W}$ represents water in MesoDyn simulation.

The interaction energies $\varepsilon_{I J}$ of the different types of segments represent the pair-wise interactions of beads in the similar way that defined in the Flory-Huggins model [45]. $\varepsilon_{I J}$ can be derived from numerous methods, such as atomistic simulation [45, 46], empirical methods [47], and experimental data [48], of which the simplest approach is based on the regular solution theory and relates the FloryHuggins parameter $\chi$ to the component solubility parameter $\delta$. The parameters can be considered to represent the nonideal interactions and the strength of repulsion interaction between different components is characterized by $\varepsilon_{\mathrm{AB}}$ $>0$, in units of kilojoules per mole. The effective meanfield interactions vary with both concentration and temperature, $\chi_{\text {eff }}=\alpha+\beta / T$, of which $\alpha$ represents the entropic contribution that is added to take into account the difference in size of solvent and solute atoms, and $\beta$ represents the enthalpic contributions.

The solvent-polymer interaction parameters are estimated as

$\chi_{\text {eff }}=\left(\delta_{I}-\delta_{J}\right)^{2} V_{\text {ref }} / \mathrm{RT}+\chi S$

where $V_{\text {ref }}$ is the monomer volume, and $\chi s$ is the entropy contribution to the mixing energy, which was neglected in this work since it is a relatively small correction in comparison to the other terms [44]. The solubility parameter $\delta$ is related to each component's volumetric cohesive energy density $E_{\mathrm{coh}} / V$ of the system via the definition [49]

$\delta=\sqrt{\frac{E_{\mathrm{coh}}}{V}}$

The values of $\delta$ can calculated directly from atomistic simulation using Eq. 3 [46].

It is known that the interaction parameters are also composition-dependent for a poly(ethylene oxide-blockpropylene oxide) solution. Bae et al. [49] used an extended Flory-Huggins equation to investigate the relationship between the parameters and concentrations for vapor-liquid or liquid-liquid equilibria at different temperatures through the vapor pressure method and the semi-empirical model. Therefore, the pair-wise interactions in MesoDyn should be considered as the effective Flory-Huggins parameters, like Baulin and Halperin's work [50], $\bar{\chi}=\chi_{\text {eff }}+f(\phi)$. From previous successful simulations for poly(ethylene oxideblock-propylene oxide) solutions [30-32, 34, 44, 45, 5052], $\chi_{\mathrm{EW}}$ and $\chi_{\mathrm{PW}}$ were selected as the values from 1.35 to 1.7 at different concentrations from atomic simulations. Obviously, the parameters should be considered as the "effect" Flory-Huggins parameters $\chi_{\text {eff. }}$ In the present simulation, the effect Flory-Huggins parameters are chosen to be $\chi_{\text {eff }}, \mathrm{EW}=1.4, \chi_{\mathrm{eff}}, \mathrm{PW}=1.7$ for solvent-polymer interaction, and $\chi_{\mathrm{eff}} \mathrm{EP}=3.0$ for polymer-polymer interaction, which are the same as our previous work $[53,54]$ and the other group's simulation [30-32, 34, 44, 45, 52, 55]. The MesoDyn input parameter $\lambda_{I J}$ is related to the effect Flory-Huggins parameters via $\lambda_{I J}=\chi_{\text {eff }, I J} \mathrm{RT}$, from which the pairs of species in the system can be obtained. They are $\lambda_{\mathrm{EW}}=3.47, \lambda_{\mathrm{PW}}=4.21$, and $\lambda_{\mathrm{EP}}=7.43 \mathrm{~kJ} / \mathrm{mol}$ at $298 \mathrm{~K}$. For all simulations, the parameters in the MesoDyn program are chosen as: the time step $\Delta \tau=50 \mathrm{~ns}$ (dimensionless time step $\Delta \tau=0.5$ ), the noise scaling parameter $\Omega=100$, the compressibility parameter $\kappa_{H}^{\prime}=25$, the grid parameter $d=\mathrm{ah}^{-1}$ $=1.1543$, and the total simulation time is 50,000 steps (i.e., $2.5 \mathrm{~ms}$ ). The simulations are performed at $298 \mathrm{~K}$ and in a cubic grid with $32 \times 32 \times 32 \mathrm{~nm}$ cells of mesh size $h$. All the simulations are carried out using the MesoDyn module in the commercial software package Material Studio 4.3, from Accelrys Inc.

The shear behavior in the MesoDyn simulation can be considered to simulation the stirring or oscillation behaviors in the experiment. In the simulation for an incompressible block polyether system, a fluctuating scalar field $\phi_{k}$ is usually added to the density function to represent the shear. It can be presented by the Fokker-Planck equation [56-58]

$\frac{\partial P}{\partial t}[\phi, t]=\int_{k} \frac{\delta}{\delta \phi_{k}}\left[\mu\left(\frac{\delta}{\delta \phi_{-k}}+\frac{\delta H[\phi]}{\delta \phi_{-k}}\right)-A \omega \cos \omega t k_{x} \frac{\partial}{\partial k_{y}} \phi_{k}\right] P[\phi, t]$

Here, $\phi_{k}$ is a fluctuating scalar field described by Landau-Brazovskii Hamiltonian, and $\mu$ is an Onsager coefficient, which is approximated by $\mu=\mu\left(k_{0}\right)$ and is assumed to be frequency independent [56]. The last term in Eq. 4 describes the coupling between the shear flow $v=A \omega \cos \omega t y e_{x}$ and the gradient of $\phi_{k}$. The effective steadystate thermodynamic potential for the reorientational transitions occurring in the hexagonal phase is obtained after some approximations [58]. The equation is

$$
\Phi_{\text {hex }}=\Phi_{\text {hex }}^{(0)}+\beta^{3} \Phi_{\text {hex }}^{(1)} F(\dot{\gamma}) \cos (6 \theta), \quad F(\dot{\gamma})= \begin{cases}-\dot{\gamma}^{2}, & \dot{\gamma} \rightarrow 0 \\ \dot{\gamma}^{-1 / 3}, & \dot{\gamma} \rightarrow \infty\end{cases}
$$

Where the angle $\theta$ determines the orientation of the hexagonal lattice $(\theta=0$ corresponds to the orientation in real space). Generally, the potential $\Phi_{h e x}^{\{\}}$is time-dependent. In this model, the minimum of $\Phi_{h e x}^{\{\}}$determines the stable orientation, and the potential $\Phi_{h e x}^{\{\}}$can be viewed as a dynamic extension of the equilibrium free energy. Using the equations above, the oriented hexagonal phase can be constructed in the MesoDyn simulation. 


\section{Results and discussion}

The effect of concentration on the morphology of block polyether aggregate

The PO groups of block polyether can form hydrophobic core of micelle when the concentration is larger than its CMC. Therefore, the isosurface of PO groups can be used to mimic the micellar morphology in the MesoDyn simulation. Figure $2 \mathrm{a}$ shows the effect of concentration on the PO-block isosurface of branched block polyether A in the $32 \times 32 \times 32 \mathrm{~nm}$ box. When the concentration is $5 \mathrm{vol} \%$, no micelles are formed within the simulation time of $2.5 \mathrm{~ms}$. However, the PO groups distribute unevenly in the box and have aggregation trend. When the concentrations are $7.5,10$, and $15 \mathrm{vol} \%$, the asymmetry micelles are observed. When the concentration is $20 \mathrm{vol} \%$, symmetry micelles are found at the end of simulation. However, the micelles are not perfectly spherical. Interestingly, the imperfect spherical micelles are formed during the simulation, which has been reported in the literatures [34, 52, 59]. When the concentration ascends to $50 \mathrm{vol} \%$, the rod-like micelles are found. While the concentration is larger than $60 \mathrm{vol} \%$, the wormlike micelles are formed.

The aggregation behavior of the linear block polyether B, which has the same PO content as A, is also investigated. Figure $2 \mathrm{~b}$ shows that the aggregation morphology of polyether $B$ changes with the increase of concentration. When the concentration is $10 \mathrm{vol} \%$, the pre-micelle can be found. While the concentration is $20 \mathrm{vol}$ $\%$, the huge micelles are formed. However, the spherical and rod-like micelles are found as the concentration ascends to $40 \mathrm{vol} \%$. Until the concentration ascends to $80 \mathrm{vol} \%$, the wormlike micelles appear. It can be found that both polyether A and B can form spherical, rod-like, wormlike micelles, and micellar clusters in aqueous solution, however, polyether A forms spherical and wormlike micelles at lower concentration than B.

The aggregation number can be calculated in the simulation to investigate the aggregation behavior of block polyethers $[34,52,55]$. In a $32 \times 32 \times 32 \mathrm{~nm}$ cubic grid, the numbers of the micelles are averaged about 60 and 50 in the 20 vol\% A and B systems, respectively. Then, the

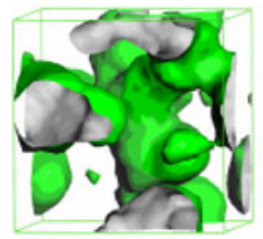

(i)

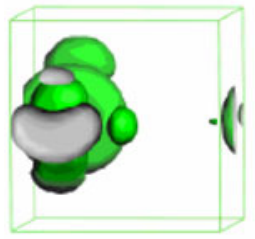

(iv)

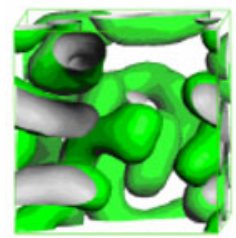

(vii)

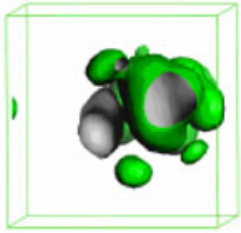

(ii)

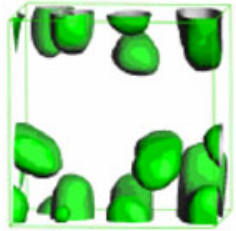

(v)

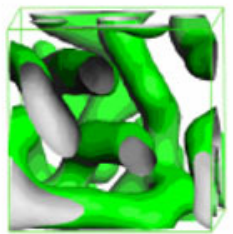

(viii)

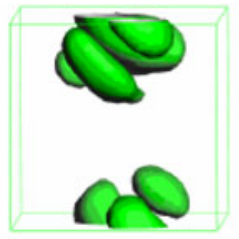

(iii)

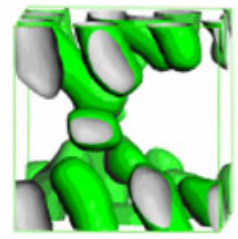

(vi)

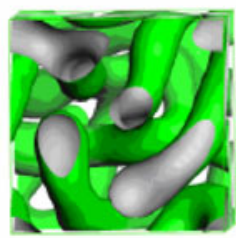

(ix)

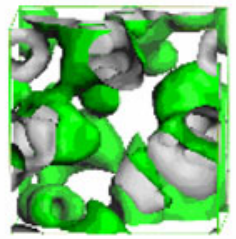

(i)

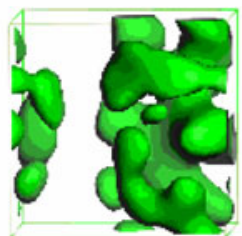

(iv)

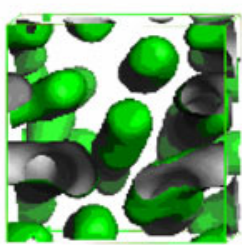

(vii)

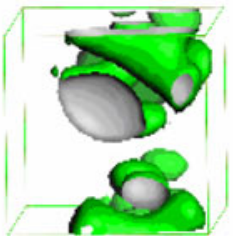

(ii)

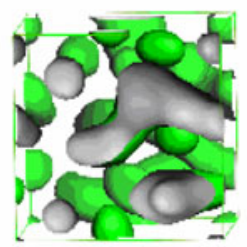

(v)

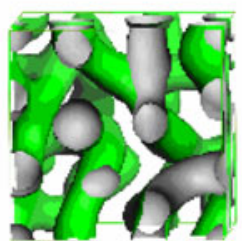

(viii)

(A)

(B)

Fig. 2 The effect of concentration on the aggregation morphology of the branched and linear block polyethers. a $i$ to $i x$ represent the concentration (vol\%) of polyether A: 5, 7.5, 10, 15, 20, 50, 60, 65,

and 80 . b $i$ to viii represent the concentration (vol\%) of polyether B: $10,20,30,35,40,50,60$, and 80 
average core radius $\left(R_{\mathrm{c}}\right)$ of $\mathrm{PO}$ monomer is calculated, which are about 5.0 and $5.4 \mathrm{~nm}$ for $20 \mathrm{vol} \% \mathrm{~A}$ and B. To a good approximation, the aggregation number $(N)$ scales linearly with the third power of the average core radius $\left(R_{\mathrm{c}}\right):[60]$

$N n V_{\mathrm{PO}}=\frac{4}{3} \pi R_{\mathrm{c}}^{3}$

Where $n=80$ and 40 , are the PO polymerization degree of $\mathrm{A}$ and $\mathrm{B}$, and the propylene oxide volume $V_{\mathrm{PO}}$ is $0.0954 \mathrm{~nm}^{3}[34,52,55]$. The $N$ values of A and B at $20 \mathrm{vol} \%$ are 72 and 170 calculated by Eq. 6. This orderliness agrees with Perreura's result in the experiment [61]. Nolan et al. have found that the aggregation number of linear triblock copolymers appeared to increase with the increasing molecular weight while keeping the PEO/PPO ratio constant [62]. However, here the branched block polyether has a smaller aggregation number than the linear polyether. Therefore, the branched structure can increase the steric interactions of PPO and $\mathrm{PEO}$ and influence the aggregation behavior greatly.
The morphology of block polyether aggregate at various simulation times

Yuan et al. has discussed the effect of time evolution on the aggregation morphology [52]. However, the detailed morphology change has not been given. Ganguly et al. found that P123 solutions showed a sphere-to-rod micellar growth with time evolution in the aqueous medium unlike the other aqueous poly(ethylene oxide-block-propylene oxide) systems by dynamic light scattering, viscometry, and smallangle neutron-scattering techniques [33]. Here, the influence of the time evolution on the morphology of polyether aggregates and the sphere-to-rod micellar growth behavior dependent on time is investigated by the MesoDyn simulation. Figure 3 a gives the variation of the aggregation morphology in the 10 vol\% A solution with the time evolution. The PO groups begin to gather and form the premicelles at $75 \mu \mathrm{s}$. A number of spherical micelles are formed at $225 \mu \mathrm{s}$. The interesting case is that an anisometric huge micelle is formed from the spherical micelles at $275 \mu$ s and then the micelle grows huger. At $750 \mu$ s the huge micelle deforms into small micelles. The rod-like micelles appear at $2 \mathrm{~ms}$ and are kept until the end

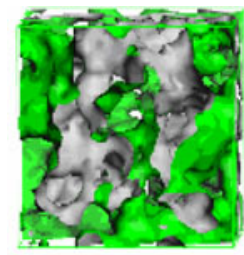

(i)

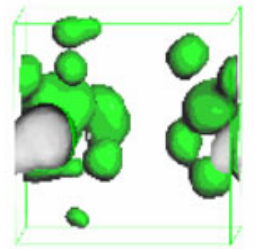

(iv)

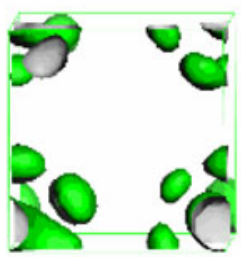

(vii)

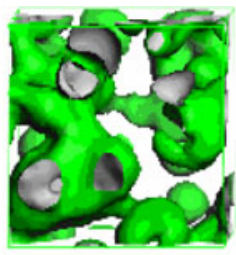

(ii)

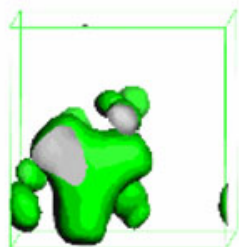

(v)

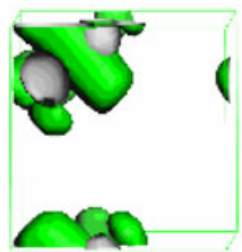

(viii)

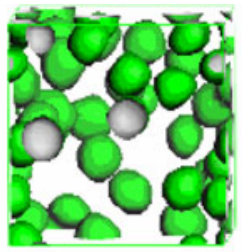

(iii)

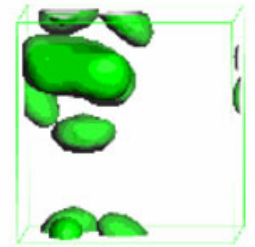

(vi)

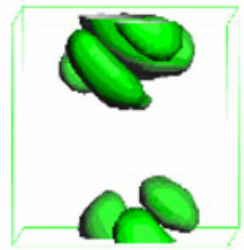

(ix)

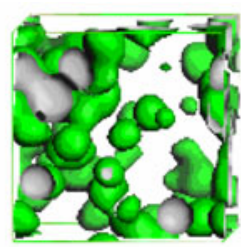

(i)

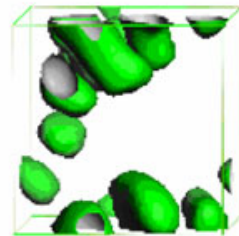

(iv)

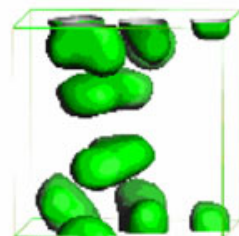

(vii)

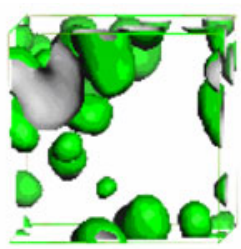

(ii)

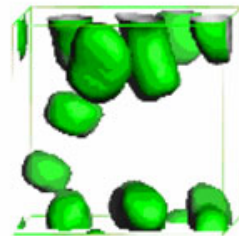

(v)

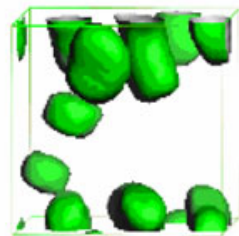

(viii)

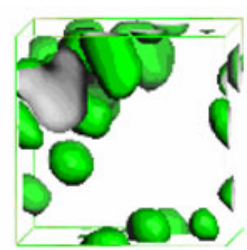

(iii)

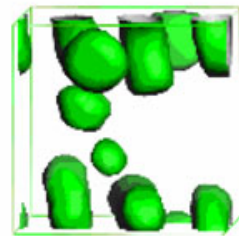

(vi)

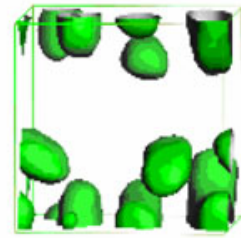

(ix)

(A)

(B)

Fig. 3 The effect of time evolution on the aggregation morphology of the branched polyether. a $10 \mathrm{vol} \%$ A system: $(i)$ to $(i x)$ represent the time step $(\mu \mathrm{s})$ of $25,75,225,275,500,750,1,500,2,000$, and 2,500. b $20 \mathrm{vol} \%$ A system: (i) to (ix) represent the time step ( $\mu$ s) of 25,50 , $75,225,500,750,1,250,2,000$, and 2,500 


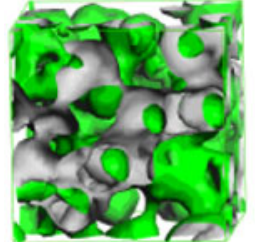

(A)

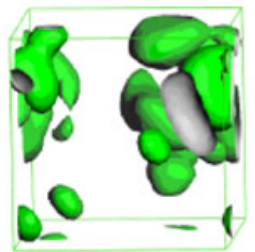

(D)

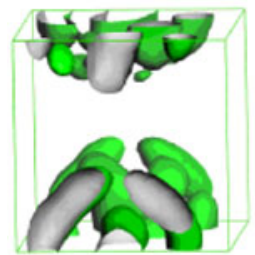

(G)

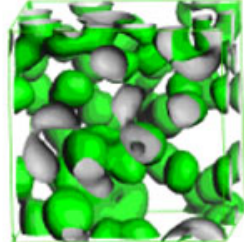

(B)

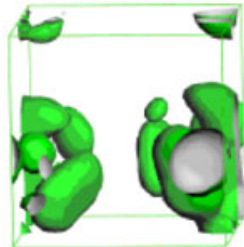

(E)

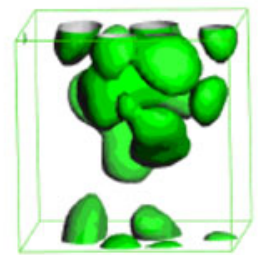

$(\mathrm{H})$

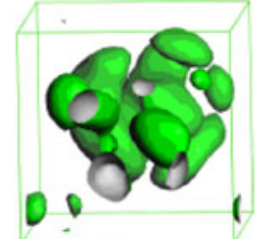

(C)

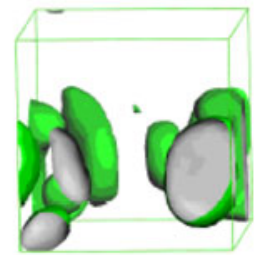

(F)

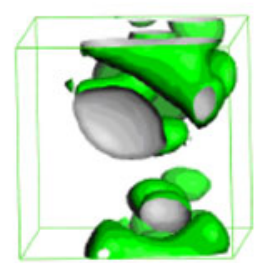

(I)
Fig. 4 The effect of time evolution on the aggregation morphology of the $20 \mathrm{vol} \%$ polyether B. a $25 \mu \mathrm{s}$, b $50 \mu \mathrm{s}$, c $250 \mu \mathrm{s}$, d $500 \mu \mathrm{s}$, e $750 \mu \mathrm{s}, \mathbf{f} 1 \mathrm{~ms}, \mathbf{g} 1.5 \mathrm{~ms}, \mathbf{h} 2 \mathrm{~ms}$, and $\mathbf{i} 2.5 \mathrm{~ms}$ of simulation. However, when the concentration of A arrives at 20 vol\% (shown in Fig. 3b), a large number of micelles and micellar clusters are formed at $25 \mu \mathrm{s}$. Then the small micelles grow into huge micelles and afterward the micelles just have a little variation in the shape of pseudosphere, which is also found in Zhao and Wanka's work of simulation and experiment [55, 63].

In order to compare the aggregation behavior between polyethers $\mathrm{A}$ and $\mathrm{B}$, the effect of time evolution on the morphology of $\mathrm{B}$ aggregate is also investigated. No micelles are formed within the simulation time of $2.5 \mathrm{~ms}$ in the $10 \mathrm{vol} \% \mathrm{~B}$ system. When the concentration arrives at $20 \mathrm{vol} \%$, the micelles are found during the simulation. Figure 4 shows the variations of the aggregation morphology in the $20 \mathrm{vol} \% \mathrm{~B}$ system with the time evolution. It is similar to the case of $10 \mathrm{vol} \%$ A that the small spherical micelles are found at the beginning of the simulation (about $50 \mu \mathrm{s})$. The small spherical micelles gather into huge micellar clusters at $250 \mu \mathrm{s}$. Then the clusters and the small spherical micelles transform to each other. At the end of simulation, the small micelles and huge micellar clusters coexist. The micellar clusters are also found for other systems in the experiment $[64,65]$ and simulation [66].

It is reputed that the micellar formation is a homeostasis process in the surfactant solution. The micelles and monomers are in the equilibrium. The exchange between micelle and monomers are too fast to observe in the experiment. However, in the MesoDyn simulation, the exchange process can be observed clearly. It can be found
Fig. 5 Time evolution of order parameters for A and B systems at different concentrations: a 10 vol $\%$ A, b 20 vol $\%$ A, c $10 \mathrm{vol} \% \mathrm{~B}$, and d $20 \mathrm{vol} \% \mathrm{~B}$
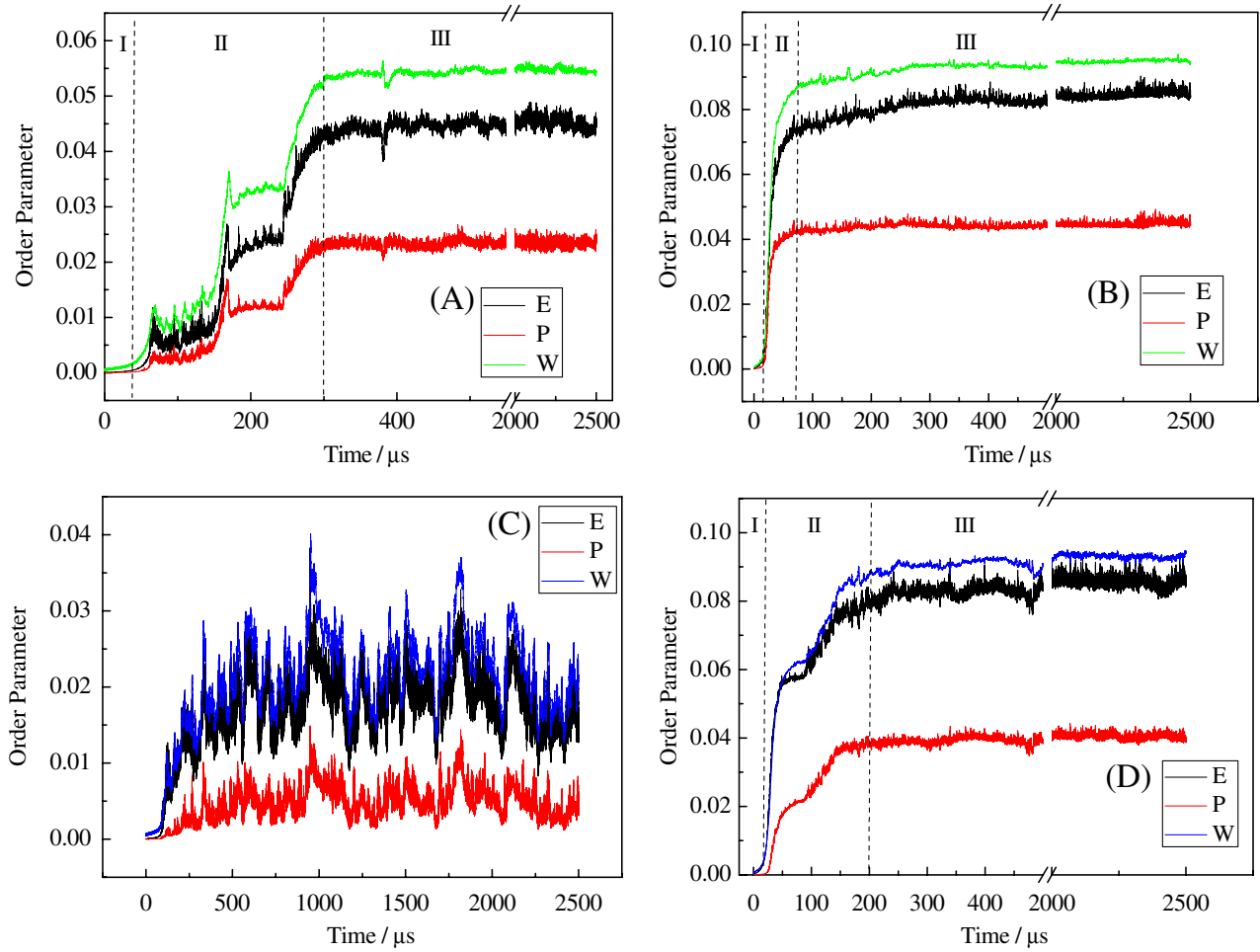


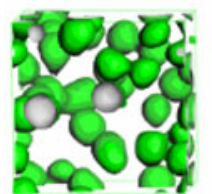

i

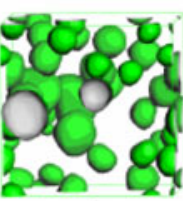

i

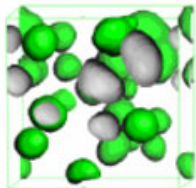

ii

(A)

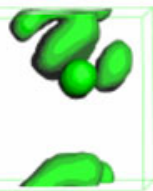

iii

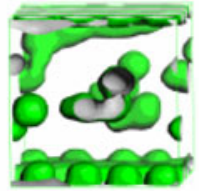

iv

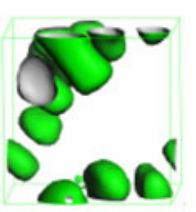

i

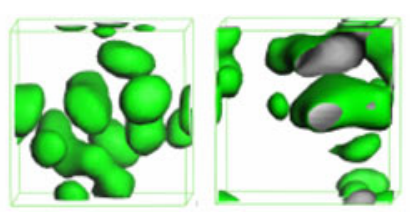

ii iii

(a)

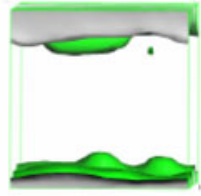

iv

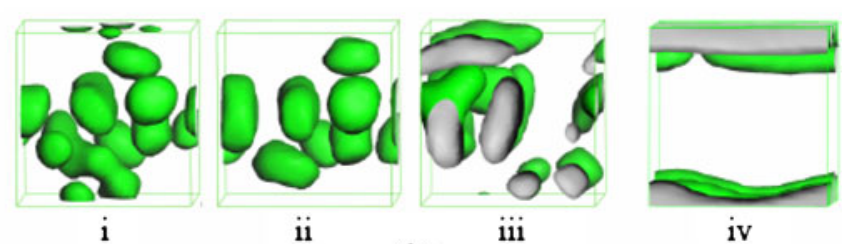

(b)

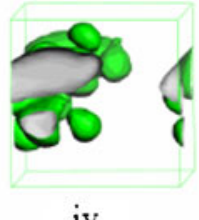

iv

(B)

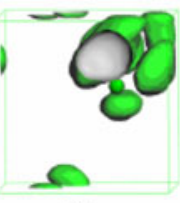

iii

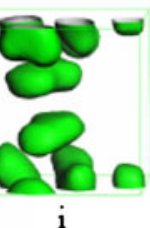

ii

( I )

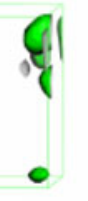

(C)

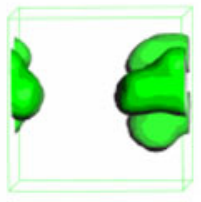

iii

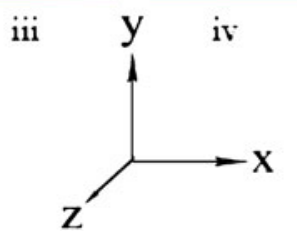

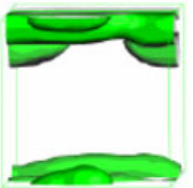

\section{$-x$}

Fig. 6 The aggregation morphology of the branched polyether at various simulation times under different shear rate. $(I)$ : a to $\mathbf{c}$ represent the morphology of $10 \mathrm{vol} \%$ polyether A at time of $0.2,0.25$, and

that the larger the concentration is the less time is needed for the micelles to form. The change of the A micelles at the concentration of $10 \mathrm{vol} \%$ with the time evolution is more obvious than that of $20 \mathrm{vol} \%$. This is because that A molecules disengage from the micelle leading to the change of micellar morphology at the concentration of $10 \mathrm{vol} \%$. While the concentration is $20 \mathrm{vol} \%$, A molecules in the form of monomers are enough in the system. When the molecules disengage from the micelle, the other molecules can rapidly supplement the micelle. Therefore, the micellar morphology of $20 \mathrm{vol} \%$ polyether A changes a little with the time evolution. The time to form micelles for $20 \mathrm{vol} \%$ $\mathrm{A}$ is shorter than that of $\mathrm{B}$. This is because that the hydrophobic effect of $\mathrm{A}$ is much stronger than that of $\mathrm{B}$ and so A micelles are easier to form.

Two breaks were observed in the surface tension isotherms of block polyethers [40,67], which happened to the branched block polyether A (shown in Figure S1). This phenomenon is usually ascribed to the broad distribution of molecular weight of macromolecules, change of molecular conformation at the air/water interface, or the formation of unimolecular micelles or oligomers before CMC [67, 68]. Meanwhile, Huang et al. found that the two breaks appearing in the surface tension isotherms of surfactant mixtures, and considered as the aggregates forming at the first break (before CMC) $[69,70]$. Here, great deals of pseudo-spherical micelles are found at $20 \mathrm{vol} \%$, but a spot of rod-like micelles are found at the concentration of $10 \mathrm{vol}$ $\%$. The formation of the rod-like micelles may cause the polyether molecules transferring from the air/water interface to the bulk solution, which can lead to the slow decrease of surface tension and the appearance of break point in the surface tension isotherm. However, the formation of the rod-like micelles at the low concentration waits to be proved in the experiment in future.

The order parameter $(p)$, which is the mean-squared deviation from homogeneity in the system representing the characteristics of phase separation and compressibility, is defined as follows:

$p \equiv \overline{\theta-\bar{\theta}^{2}}=\frac{\int_{V} \sum_{1}\left[\theta_{1}^{2}(r)-\left(\theta_{1}^{0}\right)^{2}\right] \mathrm{dr}}{V}$

Where $\theta$ and $V$ are the polymer volume fraction and cell volume, respectively. The variation of parameter $p$ during the simulation can show the process of phase separation more distinctly than the image changes of the aggregate. The variations of $p$ values with time evolution for $10 \mathrm{vol} \%$ 

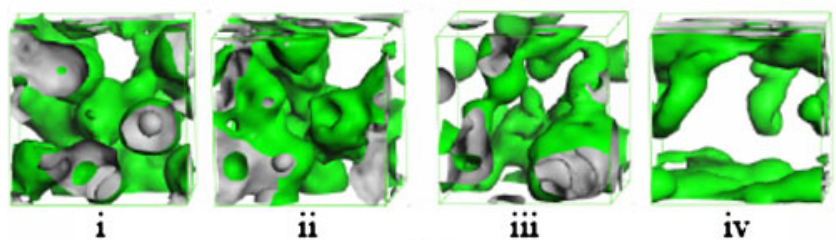

(A)
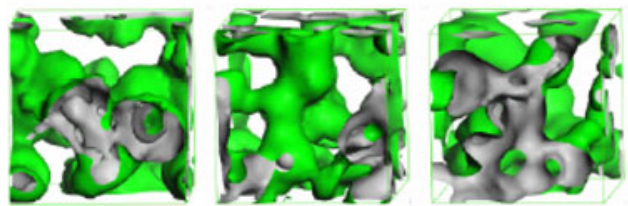

iii

(B)

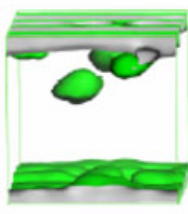

iv

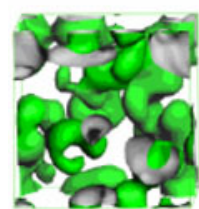

i

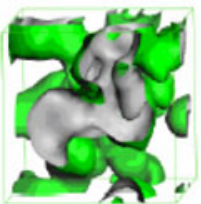

ii
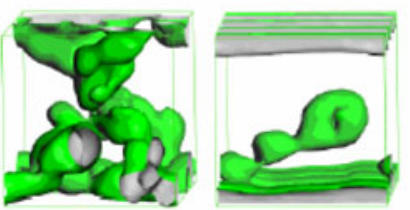

iii

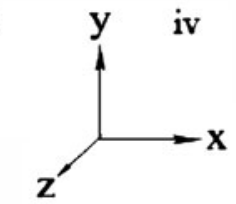

( I )

(C)

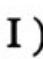

Fig. 7 The aggregation morphology of the linear polyether at various simulation times under different shear rate. $(I)$ : a to $\mathbf{c}$ represent the morphology of $10 \mathrm{vol} \%$ polyether B at time of $0.2,0.25$, and $2.0 \mathrm{~ms}$.

A are shown in Fig. 5a. The values of $p$ increase with the time evolution and three stages are found. The stage I is about 0 to $40 \mu \mathrm{s}$, during which the $p$ value and aggregation morphology change little. In this stage the system are almost homogenous. Then in stage II (about 40 to $300 \mu \mathrm{s}$ ) the $p$ value increase rapidly and the micellar morphology are found. Interestingly, in this stage, the curves of $p$ values vs. time evolution have two peaks, which means that there are aggregate transforming during the spherical micelles formation process. At last, in stage III, the $p$ values change slowly to reach the equilibrium and the system evolves slowly to get the most stable state. When the concentration of A arrives at $20 \mathrm{vol} \%$, the variations of $p$ value with the time are shown in Fig. 5b. It is clear that the stage I and II are only 20 and $60 \mu \mathrm{s}$, respectively. However, in stage II no peaks are observed during the $p$ values increasing process.

Contrasted with the branched block polyether, the variations of $p$ values of $\mathrm{B}$ at 10 and $20 \mathrm{vol} \%$ are shown in Fig. 5 c, d. When the concentration of B is $10 \mathrm{vol} \%$, no aggregate is found during the whole simulation time. The $p$ values vs. time curves are oscillating all the time and $p$ values of $\mathrm{E}$ and $\mathrm{W}$ beads are almost the same. When the concentration arrives at $20 \mathrm{vol} \%$, the micelles are found and three stages appear in the $p$ value vs. time curves. The

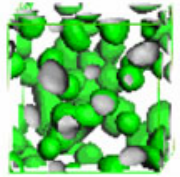

i

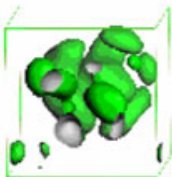

i

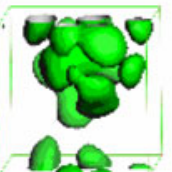

i

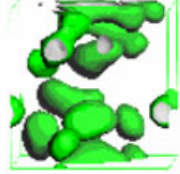

ii

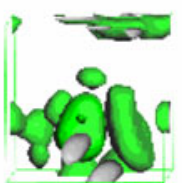

ii

(b)

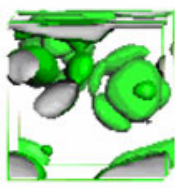

iii

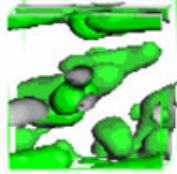

iv (a)

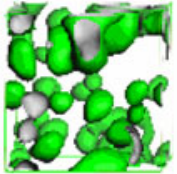

iii

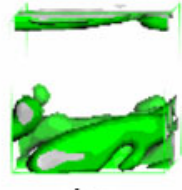

iv

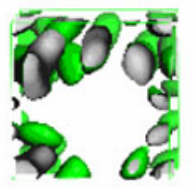

ii

(c)
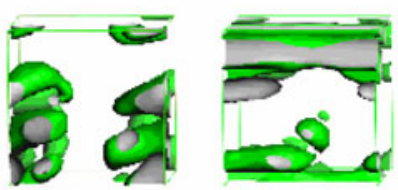

( II )

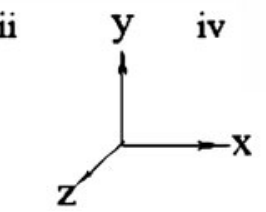

(II): a to $\mathbf{c}$ represent the morphology of $20 \mathrm{vol} \%$ polyether B at time of $0.1,0.25$, and $2.0 \mathrm{~ms}$. The shear rates of $i-i v$ are the same as Fig. 6

stages I and II continue for only 20 and $180 \mu \mathrm{s}$, respectively. The duration of the stage II for B is longer than that for $\mathrm{A}$, indicating that the micellar formation process of $A$ is faster and easier than that of the linear block polyether. Usually, the dynamic process of micellar formation is too fast to observe in the experiment. However, it can be investigated in the MesoDyn simulation. Although the duration of stage II is not the real time of the micelle formation, it can represent the formation speed by contrast $[34,55]$.

The effect of shear on the morphology of aggregate

The study on the shear dependence of the aggregation morphology of amphiphiles is important, because the stirring or ultrasonic treatment always occurs during the process of preparing the amphiphilic systems. Yuan et al. has found the hexagonal and lamellar phases present under the shear in the simulation [34]. In order to show the difference of the micellar morphology of the branched and linear block polyethers under the shear, the micellar morphology variations of $10 \mathrm{vol} \% \mathrm{~A}$ with the simulation time and at different shear rate are studied, which is shown in Fig. 6 (I). It can be seen that the spherical micelles are 

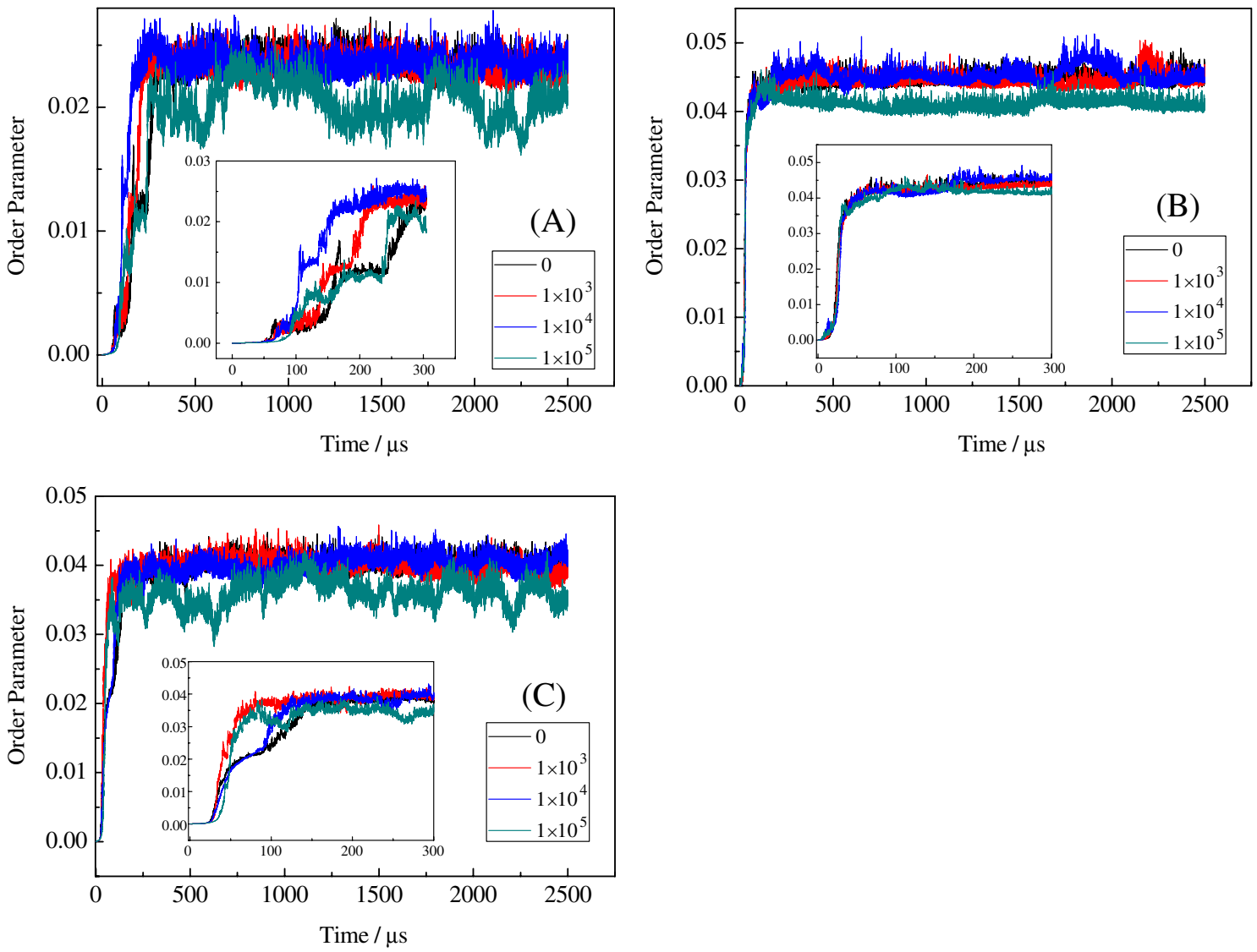

Fig. 8 Time evolution of order parameters for P beads of polyethers A and B systems at different concentrations: a 10 vol $\%$ A, b 20 vol\% A, and c $20 \mathrm{vol} \% \mathrm{~B}$

formed at $0.2 \mathrm{~ms}$ in the absence of shear. When the shear rate is $1 \times 10^{3} \mathrm{~s}^{-1}$, some of the micelles aggregate to form micellar clusters. The huge micelles are formed as the shear rate arrives at $1 \times 10^{4} \mathrm{~s}^{-1}$. However, when the shear rate arrives at $1 \times 10^{5} \mathrm{~s}^{-1}$, the huge micelles are disaggregated into spherical micelles arraying along the shearing direction $x$-axis. When the simulation time increases to $0.25 \mathrm{~ms}$, the huge micelles and micellar clusters are formed in the presence of shear. As the simulation time is $1.25 \mathrm{~ms}$, the micellar clusters aggregate to form huge micelles in the presence of shear. However, the rod-like micelles are only formed under the shear rate of $1 \times 10^{5} \mathrm{~s}^{-1}$.

When the concentration of A increases to $20 \mathrm{vol} \%$, the rod-like micelles are found at $1.25 \mathrm{~ms}$ under the shear rate of $1 \times 10^{4} \mathrm{~s}^{-1}$. When the shear rate is $1 \times 10^{5} \mathrm{~s}^{-1}$, the rod-like micelles are found at $0.2 \mathrm{~ms}$. That is, the larger the concentration of $\mathrm{A}$ is, the easier the wormlike micelles is formed under the effect of shear.

The aggregation morphology of $10 \mathrm{vol} \% \mathrm{~B}$ at different simulation time under different shear rate is shown in Fig. 7 (I). It can be seen that the PO chains have the trend of forming aggregate when the shear rate is lower than $1 \times 10^{5} \mathrm{~s}^{-1}$. The rod-like micelles are formed under the shear rate of $1 \times 10^{5} \mathrm{~s}^{-1}$. Figure 7 (II) shows that the effect of the shear on the aggregation morphology of $\mathrm{B}$ is more complex. It can be seen that the micelles are formed at $0.1 \mathrm{~ms}$ in the absence of shear, then some of the micelles aggregate to form huge micelles as the shear rate increase to $1 \times 10^{3} \mathrm{~s}^{-1}$. However, when the shear rate arrives at $1 \times 10^{4} \mathrm{~s}^{-1}$, the huge micelles are disaggregated into spherical micelles. When the shear rate increases to $1 \times 10^{5} \mathrm{~s}^{-1}$, the rod-like micelles and micellar clusters coexist. When the simulation time increases to $0.25 \mathrm{~ms}$, the rod-like and micellar clusters are found as the shear rate is larger than $1 \times 10^{3} \mathrm{~s}^{-1}$. As the simulation time is $2.0 \mathrm{~ms}$, the micellar clusters are formed in the absence of shear. When the shear rate is $1 \times 10^{3} \mathrm{~s}^{-1}$, the micellar clusters are disaggregated into ellipsoidal micelles. When the shear rate increases to $1 \times 10^{4} \mathrm{~s}^{-1}$, the ellipsoidal micelles grow and form huge micelles. At last the rod-like micelles are formed under the shear rate of $1 \times 10^{5} \mathrm{~s}^{-1}$.

It may be seen that the effects of shear on the aggregation morphology of the branched and linear block polyethers are different clearly. The PPO chains can aggregate via the hydrophobic effect. When the shear is present in the system, the micelles can be distorted and 
formed rod-like micelles by the external force. Meanwhile, the disaggregation of the micellar clusters under the external force appears especially in the $20 \mathrm{vol} \% \mathrm{~B}$ system. This indicates that the huge micelles or micellar clusters of the branched polyether are difficult to distort because A molecule can be coexist in different micelles and the interactions between the micelles are stronger due to the branched structure of the molecules. It can also be found that the formation of the rod-like micelles in $20 \mathrm{vol} \% \mathrm{~A}$ system needs more time than that in B system, which can also indicate that the interaction between $\mathrm{A}$ micelles is stronger than that of $\mathrm{B}$ because of the tangle of the branched A chains.

In order to investigate the effect of shear on the aggregation morphology during the whole process of simulation, the $p$ values of $\mathrm{P}$ beads at different shear rate are shown in Fig. 8. It can be seen from Fig. 8a that the effect of shear on the micellar formation is strong at the initial stage of the simulation, which means that the shear can induce the formation of the micelles at the lower shear rate, however, slow down the micellar formation at the higher shear rate $\left(1 \times 10^{5} \mathrm{~s}^{-1}\right)$. When the micelles are formed, the $p$ values oscillate with the time evolution under the shear. However, when the A concentration increases to $20 \mathrm{vol} \%$, the effect of the shear in not clear at the initial stage (Fig. 8b). This is because that the hydrophobic interaction increases with the increase of the PPO groups and the micelles form in less than $100 \mu$ s, the additional external force cannot affect on the formation of the micelles. However, the effect of the shear on the formation of micelles at the initial stage is clear in the $20 \mathrm{vol} \% \mathrm{~B}$ system (Fig. 8c). It is known that the rod-like micelles can form at the shear rate of $1 \times 10^{5} \mathrm{~s}^{-1}$. It can also be found that at the anaphase of the simulation the oscillation of the parameter $p$ for the $\mathrm{B}$ system with the time evolution is more frequent than that of $\mathrm{A}$ at the shear rate of $1 \times 10^{5} \mathrm{~s}^{-1}$. However, the oscillation period is long for the A system. This means that the micelles of $\mathrm{B}$ is easier to disaggregate and form than that of $\mathrm{A}$ in the presence of shear, implying that the hydrophobic effect of $\mathrm{A}$ is stronger than that of $\mathrm{B}$.

\section{Conclusions}

The different aggregation behaviors of the branched block polyether $\mathrm{A}$ and linear $\mathrm{B}$ were investigated by the MesoDyn method. Both A and B polyethers can form the spherical, rod-like, wormlike micelles, and micellar clusters in aqueous solution. However, polyether $\mathrm{A}$ can form micelles at a lower concentration and have a smaller aggregation number than B. The MesoDyn simulation can provide the information of the time-dependent growth behavior. Both the polyethers show the time-dependent micellar growth behavior. The concentration and the structure of the block polyether can influence the micellar morphology. A and B polyethers show different shape transition with the time evolution. When the shear rate is $1 \times 10^{5} \mathrm{~s}^{-1}$, the shear can induce the sphere-to-rod transition of both block polyethers micelles. When the shear rate is lower than $1 \times 10^{5} \mathrm{~s}^{-1}$, the effect of shear on the shape variation of both polyethers micelles is different. The shear can induce branched molecules form huge micelles and micellar clusters, while for the linear block polyether the huge micelles form and then disaggregate with the time evolution.

Acknowledgments The authors gratefully acknowledge the financial support from the Natural Science Foundation of China (20573067, 20873077) and Key Laboratory of Microgravity, Institute of Mechanics, Chinese Academy of Sciences.

\section{References}

1. Alexandridis P, Athanassiou V, Hatton TA (1995) Pluronic-p105 PEO-PPO-PEO block copolymer in aqueous urea solutions: micelle formation, structure, and microenvironment. Langmuir 11(7):2442-2450

2. Alexandridis P, Hatton TA (1995) Poly (ethylene oxide)-poly (propylene oxide)-poly (ethylene oxide) block copolymer surfactants in aqueous solutions and at interfaces: thermodynamics, structure, dynamics, and modeling. Colloids Surf, A 96(1-2):1-46

3. Alexandridis P, Nivaggioli T, Hatton TA (1995) Temperature effects on structural properties of pluronic p104 and f108 PEO-PPO-PEO block copolymer solutions. Langmuir 11(5):1468-1476

4. Liang X, Guo C, Ma J, Wang J, Chen S, Liu H (2007) Temperature-dependent aggregation and disaggregation of poly (ethylene oxide)-poly(propylene oxide)-poly(ethylene oxide) block copolymer in aqueous solution. J Phys Chem B 111 (46): $13217-13220$

5. Yang B, Guo C, Chen S, Ma J, Wang J, Liang X, Zheng L, Liu H (2006) Effect of acid on the aggregation of poly(ethylene oxide)poly(propylene oxide)-poly(ethylene oxide) block copolymers. J Phys Chem B 110(46):23068-23074

6. Firestone MA, Tiede DM, Seifert S (2000) Magnetic field-induced ordering of a polymer-grafted biomembrane-mimetic hydrogel. J Phys Chem B 104(11):2433-2438

7. Firestone MA, Wolf AC, Seifert S (2003) Small-angle X-ray scattering study of the interaction of poly(ethylene oxide)-b-poly (propylene oxide)-b-poly(ethylene oxide) triblock copolymers with lipid bilayers. Biomacromolecules 4(6):1539-1549

8. Liu L-Z, Wan Q, Liu T, Hsiao BS, Chu B (2002) Salt-induced polymer gelation and formation of nanocrystals in a polymer-salt system. Langmuir 18(26):10402-10406

9. Wu C, Liu T, Chu B, Schneider DK, Graziano V (1997) Characterization of the PEO-PPO-PEO triblock copolymer and its application as a separation medium in capillary electrophoresis. Macromolecules 30(16):4574-4583

10. Alexandridis P, Holzwarth JF (1997) Differential scanning calorimetry investigation of the effect of salts on aqueous solution properties of an amphiphilic block copolymer (poloxamer). Langmuir 13(23):6052-6074

11. Michels B, Watona G, Zanab R (2001) Evidence of micelle growth in aqueous solutions of the amphiphilic poly(ethylene 
oxide)-poly(propylene oxide)-poly(ethylene oxide) triblock copolymers from differential scanning microcalorimetry. Colloids Surf A 183-185:55-65

12. Pattersona I, Chowdhryb BZ, Leharne S (1996) Evaluation of surfactant monomer concentrations in equilibrium with micelles and their temperature dependence for oxyethylene/oxypropylene/ oxyethylene triblock copolymers using dsc. Colloids Surf A 111 (3):213-222

13. Eiser E, Molino F, Porte G, Diat O (2000) Nonhomogeneous textures and banded flow in a soft cubic phase under shear. Phys Rev E 61(6):6759-6764

14. King SM, Heenan RK, Cloke VM, Washington C (1997) Neutron scattering from a poly(oxyethylene)-poly(oxypropylene)-poly (oxyethylene) copolymer in dilute aqueous solution under shear flow. Macromolecules 30(20):6215-6222

15. Pedersen JS, Svaneborgb C (2002) Scattering from block copolymer micelles. Curr Opin Colloid Interface Sci 7(3,4):158166

16. Chen SH, Mallamace F, Faraone A, Gambadauro P, Lombardo D, Chen WR (2002) Observation of a re-entrant kinetic glass transition in a micellar system with temperature-dependent attractive interaction. Eur Phys J E 9(3):283-286

17. Jorgensen EB, Hvidt S, Brown W, Schillen K (1997) Effects of salts on the micellization and gelation of a triblock copolymer studied by rheology and light scattering. Macromolecules 30 (8):2355-2364

18. Liu Y, Chen S-H, Huang JS (1998) Light-scattering studies of concentrated copolymer micellar solutions. Macromolecules 31 (18):6226-6233

19. Chen ZR, Issaian AM, Kornfield JA, Smith SD, Grothaus JT, Satkowski MM (1997) Dynamics of shear-induced alignment of a lamellar diblock: a rheo-optical, electron microscopy, and X-ray scattering study. Macromolecules 30(23):7096-7114

20. Liu YC, Chen SH, Huang JS (1996) Relationship between the microstructure and rheology of micellar solutions formed by a triblock copolymer surfactant. Phys Rev E 54(2):1698-1708

21. Schmidt G, Richtering W, Lindner P, Alexandridis P (1998) Shear orientation of a hexagonal lyotropic triblock copolymer phase as probed by flow birefringence and small-angle light and neutron scattering. Macromolecules 31(7):2293-2298

22. Waton G, Michels B, Steyer A, Schosseler F (2004) Shearinduced demixing and shear-banding instabilities in dilute triblock copolymer solutions. Macromolecules 37(6):2313-2321

23. Espanol P, Warren P (1995) Statistical mechanics of dissipative particle dynamics. Europhys Lett 30(4):191-196

24. Groot RD, Madden TJ (1998) Dynamic simulation of diblock copolymer microphase separation. J Chem Phys 108(20):87138724

25. Groot RD, Warren PB (1997) Dissipative particle dynamics: bridging the gap between atomistic and mesoscopic simulation. J Chem Phys 107(11):4423-4435

26. Fraaije JGEM (1993) Dynamic density functional theory for microphase separation kinetics of block copolymer melts. J Chem Phys 99(11):9202-9212

27. Maurits NM, Altevogt P, Evers OA, Fraaije JGEM (1996) Simple numerical quadrature rules for gaussian chain polymer density functional calculations in $3 \mathrm{~d}$ and implementation on parallel platforms. Comput Theor Polym Sci 6(1-2):1-8

28. Maurits NM, van Vlimmeren BAC, Fraaije JGEM (1997) Mesoscopic phase separation dynamics of compressible copolymer melts. Phys Rev E 56(1):816-825

29. Warren PB (1998) Dissipative particle dynamics. Curr Opin Colloid Interface Sci 3(6):620-624

30. Fraaije JGEM, Sevink GJA (2003) Model for pattern formation in polymer surfactant nanodroplets. Macromolecules 36(21):78917893
31. Fraaije JGEM, van Vlimmeren BAC, Maurits NM, Postma M, Evers OA, Hoffmann C, Altevogt P, Goldbeck-Wood G (1997) The dynamic mean-field density functional method and its application to the mesoscopic dynamics of quenched block copolymer melts. J Chem Phys 106(10):4260-4269

32. Lyakhova KS, Zvelindovsky AV, Sevink GJA, Fraaije JGEM (2003) Inverse mapping of block copolymer morphologies. J Chem Phys 118(18):8456-8459

33. Ganguly R, Kumbhakar M, Aswal VK (2009) Time dependent growth of the block copolymer p123 micelles near cloud point: employing heat cycling as a tool to form kinetically stable wormlike micelles. J Phys Chem B 113(28):9441-9446

34. Zhang X, Yuan S, Wu J (2006) Mesoscopic simulation on phase behavior of ternary copolymeric solution in the absence and presence of shear. Macromolecules 39(19):6631-6642

35. Gong H, Xu G, Ding H, Shi X, Tan Y (2009) Aggregation behavior of block polyethers with branched structure at air/water surface. Eur Polym J 45(9):2540-2548

36. Wang F, Xu G, Zhang Z, Xin X (2006) Synthesis of monodisperse cds nanospheres in an inverse microemulsion system formed with a dendritic polyether copolymer. Eur J Inorg Chem 2006(1):109114

37. Zhang Z, Xu G, Wang F, Dong S, Chen Y (2005) Demulsification by amphiphilic dendrimer copolymers. J Colloid Interface Sci 282 (1): $1-4$

38. Zhang Z, Xu G, Wang F, Dong S, Li Y (2004) Characterization and demulsification of poly(ethylene oxide)-block-poly(propylene oxide)-block-poly (ethylene oxide) copolymers. J Colloid Interface Sci 277(2):464-470

39. Xin X, Xu G, Zhao T, Zhu Y, Shi X, Gong H, Zhang Z (2008) Dispersing carbon nanotubes in aqueous solutions by a starlike block copolymer. J Phys Chem C 112(42):16377-16384

40. Xin X, Xu G, Zhang Z, Chen Y, Wang F (2007) Aggregation behavior of star-like PEO-PPO-PEO block copolymer in aqueous solution. Eur Polym J 43(7):3106-3111

41. Xin X, Xu G, Wang Y, Mao H, Zhang Z (2008) Interaction between star-like block copolymer and sodium oleate in aqueous solutions. Eur Polym J 44(10):3246-3255

42. Li YM, Xu GY, Chen AM, Yuan SL, Cao XR (2005) Aggregation between xanthan and nonyphenyloxypropyl b-hydroxyltrimethylammonium bromide in aqueous solution: MesoDyn simulation and binding isotherm measurement. J Phys Chem B 109 (47):22290-22295

43. Bai G, Nichifor M, Lopes A, Bastos M (2004) Thermodynamic characterization of the interaction behavior of a hydrophobically modified polyelectrolyte and oppositely charged surfactants in aqueous solution: effect of surfactant alkyl chain length. J Phys Chem B 109(1):518-525

44. van Vlimmeren BAC, Maurits NM, Zvelindovsky AV, Sevink GJA, Fraaije JGEM (1999) Simulation of 3d mesoscale structure formation in concentrated aqueous solution of the triblock polymer surfactants (ethylene oxide) ${ }_{13}$ (propylene oxide) ${ }_{30}$ (ethylene oxide $)_{13}$ and (propylene oxide) ${ }_{19}(\text { ethylene oxide) })_{33}$ (propylene oxide) ${ }_{19}$. Application of dynamic mean-field density functional theory. Macromolecules 32(3):646-656

45. Lam Y-M, Goldbeck-Wood G (2003) Mesoscale simulation of block copolymers in aqueous solution: parameterisation, micelle growth kinetics and the effect of temperature and concentration morphology. Polymer 44(12):3593-3605

46. Zhang M, Choi P, Sundararaj U (2003) Molecular dynamics and thermal analysis study of anomalous thermodynamic behavior of poly (ether imide)/polycarbonate blends. Polymer 44(6):19791986

47. Honeycutt JD (1998) A general simulation method for computing conformational properties of single polymer chains. Comput Theor Polym Sci 8(1-2):1-8 
48. Wescott JT, Qi Y, Subramanian L, Capehart TW (2006) Mesoscale simulation of morphology in hydrated perfluorosulfonic acid membranes. J Chem Phys 124(13):134702-134714

49. Bae YC, Shim JJ, Soane DS, Prausnitz JM (1993) Representation of vapor-liquid and liquid-liquid equilibria for binary systems containing polymers: applicability of an extended Flory-Huggins equation. J Appl Polym Sci 47(7):1193-1206

50. Baulin VA, Halperin A (2002) Concentration dependence of the flory parameter within two-state models. Macromolecules 35 (16):6432-6438

51. Horvat A, Lyakhova KS, Sevink GJA, Zvelindovsky AV, Magerle R (2004) Phase behavior in thin films of cylinder-forming aba block copolymers: mesoscale modeling. J Chem Phys 120 (2):1117-1126

52. Yuan S, Zhang X, Chan K (2009) Effects of shear and charge on the microphase formation of 123 polymer in the sba- 15 synthesis investigated by mesoscale simulations. Langmuir 25(4):20342045

53. Cao X, Xu G, Li Y, Zhang Z (2005) Aggregation of poly(ethylene oxide)-poly(propylene oxide) block copolymers in aaqueous solution: DPD simulation study. J Phys Chem A 109(45):10418 10423

54. Li Y, Xu G, Zhu Y, Wang Y, Gong H (2009) Aggregation behavior of pluronic copolymer in the presence of surfactant: mesoscopic simulation. Colloids Surf, A 334(1-3):124-130

55. Zhao Y, Chen X, Yang C, Zhang G (2007) Mesoscopic simulation on phase behavior of pluronic p123 aqueous solution. J Phys Chem B 111(50):13937-13942

56. Fredrickson GH, Helfand E (1987) Fluctuation effects in the theory of microphase separation in block copolymers. J Chem Phys 87(1):697-705

57. Morozov AN, Fraaije JGEM (2002) Orientations of the lamellar phase of block copolymer melts under oscillatory shear flow. Phys Rev E 65(3):031803

58. Morozov AN, Zvelindovsky AV, Fraaije JGEM (2000) Orientational phase transitions in the hexagonal phase of a diblock copolymer melt under shear flow. Phys Rev E 61(4):4125-4132

59. Guo SL, Hou TJ, Xu XJ (2002) Simulation of the phase behavior of the $(\mathrm{eo})_{13}(\mathrm{po})_{30}(\mathrm{eo})_{13}$ (pluronic 164)/water/p-xylene system using MesoDyn. J Phys Chem B 106(43):11397-11403
60. Mortensen K, Pedersen JS (1993) Structural study on the micelle formation of poly(ethylene oxide)-poly(propylene oxide)-poly (ethylene oxide) triblock copolymer in aqueous solution. Macromolecules 26(4):805-812

61. Perreura C, Habasa J-P, Lappb A, Peyrelasse J (2006) Salt influence upon the structure of aqueous solutions of branched PEO-PPO-PEO copolymers. Polymer 47(3):841-848

62. Nolan SL, Phillips RJ, Cotts PM, Dungan SR (1997) Light scattering study on the effect of polymer composition on the structural properties of PEO-PPO-PEO micelles. J Colloid Interface Sci 191(2):291-302

63. Wanka G, Hoffmann H, Ulbricht W (1994) Phase diagrams and aggregation behavior of poly (oxyethylene)-poly (oxypropylene) poly( oxyethylene) triblock copolymers in aqueous solutions. Macromolecules 27(15):4145-4159

64. Ganguly R, Choudhury N, Aswal VK, Hassan PA (2008) Pluronic 164 micelles near cloud point: investigating the role of micellar growth and interaction in critical concentration fluctuation and percolation. J Phys Chem B 113(3):668-675

65. Kositza MJ, Bohne C, Alexandridis P, Hatton TA, Holzwarth JF (1999) Dynamics of micro- and macrophase separation of amphiphilic block-copolymers in aqueous solution. Macromolecules 32(17):5539-5551

66. Yang S, Yuan S, Zhang X, Yan Y (2008) Phase behavior of triblock copolymers in solution: mesoscopic simulation study. Colloids Surf, A 322(1-3):87-96

67. Alexandridis P, Athanassiou V, Fukuda S, Hatton TA (1994) Surface activity of poly(ethylene oxide)-block-poly(propylene oxide)-block-poly(ethylene oxide) copolymers. Langmuir 10 (8):2604-2612

68. De Lisi R, Milioto S (2000) Poly(ethylene oxide) ${ }_{13}$-poly(propylene oxide) $)_{30}$-poly(ethylene oxide) $)_{13}$ electrolyte interactions in aqueous solutions at some temperatures. Langmuir 16(13):5579-5583

69. Han F, He X, Huang J, Li Z, Wang Y, Fu H (2004) Surface properties and aggregates in the mixed systems of bolaamphiphiles and their oppositely charged conventional surfactants. J Phys Chem B 108(17):5256-5262

70. Yan Y, Huang J, Li Z, Han F, Ma J (2003) Aggregates transition depending on the concentration in the cationic bolaamphiphile/sds mixed systems. Langmuir 19(3):972-974 